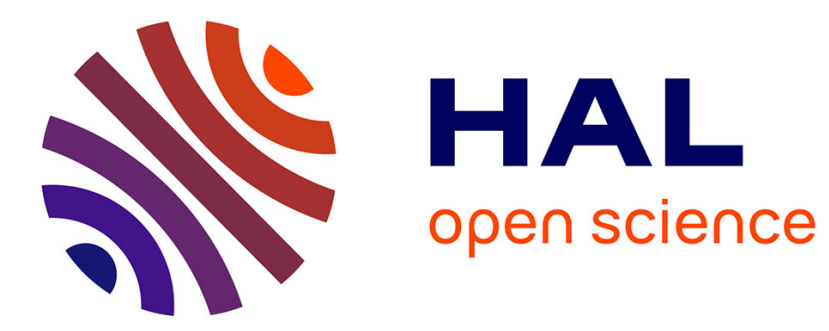

\title{
On the dispersive nature of the power dissipated into a lossy half-space close to a radiating source
}

\author{
Andrea Cozza, Benoit Derat
}

\section{To cite this version:}

Andrea Cozza, Benoit Derat. On the dispersive nature of the power dissipated into a lossy halfspace close to a radiating source. IEEE Transactions on Antennas and Propagation, 2009, 57 (9), pp.2572-2582. 10.1109/TAP.2009.2027143 . hal-00444478

\section{HAL Id: hal-00444478 \\ https://hal.science/hal-00444478}

Submitted on 8 Nov 2010

HAL is a multi-disciplinary open access archive for the deposit and dissemination of scientific research documents, whether they are published or not. The documents may come from teaching and research institutions in France or abroad, or from public or private research centers.
L'archive ouverte pluridisciplinaire HAL, est destinée au dépôt et à la diffusion de documents scientifiques de niveau recherche, publiés ou non, émanant des établissements d'enseignement et de recherche français ou étrangers, des laboratoires publics ou privés. 


\title{
On the Dispersive Nature of the Power Dissipated into a Lossy Half-Space Close to a Radiating Source
}

\author{
Andrea Cozza, Member, IEEE, and Benoît Derat, Member, IEEE
}

\begin{abstract}
In this paper, we study how the power dissipated into a lossy medium excited by a nearby antenna is affected by drifts in the electrical parameters of the lossy medium. The statistical distribution of the sensitivity of the dissipated power is determined by means of a spectral analysis of the transmission of electromagnetic energy from air into the lossy half-space. A clear link is drawn between the reactive content of the field excited by the source and the dispersiveness of the sensitivity. The case of a stratified structure is also addressed, by defining a modification factor representing the alteration of the transmissivity and of its sensitivity when a buffer layer is introduced. All of the results provided point out that, in general, the sensitivity of the total amount of power dissipated into the half-space cannot be predicted independently from a precise knowledge of the source characteristics, unless under a paraxial propagation approximation or in a far-field configuration.
\end{abstract}

Index Terms-Antennas, Lossy Media, Near Field, PlaneWave Spectrum, Sensitivity Analysis, Specific Absorption Rate, Stratified Media.

\section{INTRODUCTION}

In many practical configurations antennas stand near to a lossy half-space; an example of historical importance is that of radiating sources placed over a lossy soil [1]. Many other applications involve a similar scenario: radio-frequency hyperthermia [2] and Specific Absorption-Rate (SAR) assessment [3] are but two examples. All of these configurations share a common concern, that of being able to estimate the amount of power that will be dissipated into the lossy medium. In the context of the present discussion, we will refer to the concept of Total Dissipate Power (TDP). Depending on the actual application, the TDP needs to be maximized or minimized, but all the same its value closely depends on the electrical properties of the concerned medium, namely the electric conductivity $\sigma$ and the relative permittivity $\epsilon_{r}$. The basic need of a proper estimation of these parameters thus appears as a potential difficulty in the computation of the TDP; hence, it is fundamental to investigate how the TDP can be affected by a variation of the electrical parameters of the medium, be that due to a drift in time or rather to an uncertainty in their knowledge.

The primary aim of this paper is twofold: 1) to provide a model capable of predicting the sensitivity of the TDP

A. Cozza is with SUPELEC, Département de Recherche en Électromagnétism, Gif-sur-Yvette, France, e-mail: andrea.cozza@supelec.fr.

B. Derat is with Field-Imaging, Meudon, France, e-mail: benoit.derat@field-imaging.com.

Manuscript received ..., 2008; revised ...

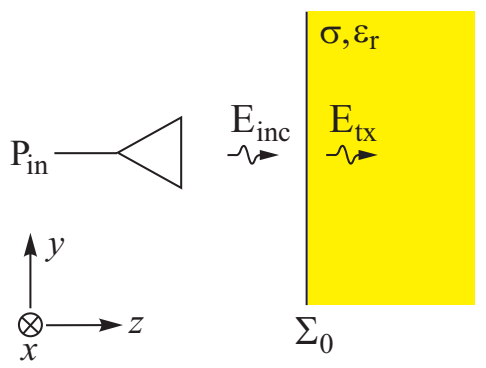

Fig. 1. The planar configuration used for the spectral analysis.

with respect to the lossy half-space characteristics for a given source and 2) to prove that the TDP sensitivity is statistically dispersed with respect to the characteristics of the source, hence leading to a non-negligible uncertainty. In particular, in the context of SAR assessment, available sensitivity analyses are very limited in scope, addressing such canonic sources as dipoles [4] and normally impinging plane waves, which are not always representative of actual ones. Although the proposed approach is based on a closed-form analysis, the results presented here do not assume a far-field configuration for the radiation source, which is a key point for their practical use.

\section{TRANSMISSION THROUGH DIELECTRIC INTERFACES}

The scenario here considered is briefly depicted in Fig. 1. A planar interface $\Sigma_{0}$ separates two half-spaces, one containing a radiating antenna and the other one a lossy homogeneous dielectric. The first half-space is assumed to be air, but any other non-magnetic medium could be considered, whereas the second one is characterized by the electric parameters $\sigma$ and $\epsilon_{r}$, which can be summed up by means of the complex permittivity $\tilde{\epsilon}=\epsilon_{r}-\mathrm{j} \sigma /\left(\omega \epsilon_{0}\right)$.

Rather than working in the space domain, it is convenient to introduce a Plane-Wave Expansion (PWE), which allows to represent the electric field distribution over a plane as a superposition of plane waves or Plane-Wave Spectrum (PWS). Considering the electric field $\mathbf{E}(\mathbf{R})$ over a plane, its PWS $\hat{\mathbf{E}}(\mathbf{K})$ is defined as [5]:

$$
\hat{\mathbf{E}}(\mathbf{K}, 0)=\int_{\Sigma_{0}} \mathbf{E}(\mathbf{R}, 0) \mathrm{e}^{\mathrm{j} \mathbf{K} \cdot \mathbf{R}} \mathrm{d} \mathbf{R},
$$

where $\mathbf{R}=x \hat{x}+y \hat{y}$ spans the entire field topography over the plane $\Sigma_{0}$ and $\mathbf{K}=k_{x} \hat{x}+k_{y} \hat{y}$ is the spectral variable; 
hereafter, capital hatted quantities will stand for PWSs. The rationale for this approach is that in the spectral domain it is much easier to represent the propagation of complex field topographies through stratified media. The PWS over planes at other distances $z$ at the right of $\Sigma_{0}$ can be easily computed introducing the propagator $P(\mathbf{K}, z)=\exp \left(-\mathrm{j} k_{z} z\right)$ :

$$
\hat{\mathbf{E}}(\mathbf{K}, z)=\hat{\mathbf{E}}(\mathbf{K}, 0) P(\mathbf{K}, z),
$$

with $k_{z}=\sqrt{k^{2}-\|\mathbf{K}\|^{2}}$ as the longitudinal propagation constant, and $k$ the propagation constant for the considered medium. The norms used throughout this work are to be intended as $\mathrm{L}^{2}$ norms applied to complex vectors, unless explicitly declared otherwise.

The PWE formalism can be defined just over the tangential components of the vectors involved; for this reason, we will decompose the electric field PWS as

$$
\hat{\mathbf{E}}=\hat{\mathbf{E}}^{\|}+\hat{E}_{z} \hat{z}
$$

having defined the tangential part of the PWS as $\hat{\mathbf{E}}^{\|}=$ $\hat{E}_{x} \hat{x}+\hat{E}_{y} \hat{y}$. The overall PWS $\hat{\mathbf{E}}$ can be derived by recalling that Gauss law in a homogeneous medium in absence of local sources reads as $\mathbf{k} \cdot \hat{\mathbf{E}}=0$, i.e.,

$$
\hat{E}_{z}=-\frac{\mathbf{K} \cdot \hat{\mathbf{E}}^{\|}}{k_{z}},
$$

where $\mathbf{k}=\mathbf{K}+k_{z} \hat{z}$ is the propagation vector for a plane wave. Thus, (3) can be recast as

$$
\hat{\mathbf{E}}=\left[\begin{array}{cc}
1 & 0 \\
0 & 1 \\
-\frac{k_{x}}{k_{z}} & -\frac{k_{y}}{k_{z}}
\end{array}\right] \hat{\mathbf{E}}^{\|}=\mathbf{T} \hat{\mathbf{E}}^{\|}
$$

Let us now consider the interface $\Sigma_{0}$ : for each plane wave impinging from the left side, there will be a transmitted plane wave. Imposing the continuity of the tangential components of the electric field through the air-dielectric interface, the PWS $\hat{\mathbf{E}}_{\text {inc }}^{\|}(\mathbf{K})$ of the incident electric field over $\Sigma_{0}^{-}$(i.e., on the air side) and the PWS $\hat{\mathbf{E}}_{\mathrm{tx}}^{\|}(\mathbf{K})$ of the field over $\Sigma_{0}^{+}$(on the right side) are related by [5]:

$$
\hat{\mathbf{E}}_{\mathrm{tx}}^{\|}(\mathbf{K})=\boldsymbol{\Pi}_{0}(\mathbf{K}) \hat{\mathbf{E}}_{\mathrm{inc}}^{\|}(\mathbf{K}),
$$

where $\boldsymbol{\Pi}_{0}(\mathbf{K})$ is the spectral transmission operator for a planar interface, as recalled in Appendix I. Hereafter, the $\mathbf{R}$ and $\mathbf{K}$ dependency of, respectively, the spatial and spectral functions will be omitted most of the time for the sake of simplicity.

The TDP is related to the transmitted field by

$$
\mathrm{TDP}=\sigma \int_{\Omega}\left\|\mathbf{E}_{\mathrm{tx}}(\mathbf{R}, z)\right\|^{2} \mathrm{~d} V,
$$

where $\Omega$ is the volume occupied by the lossy half-space. Thanks to Parseval theorem (as applied over $\Sigma_{0}^{+}$), we can state that

$$
\mathrm{TDP}=\sigma \int \frac{\left\|\hat{\mathbf{E}}_{\mathrm{tx}}\right\|^{2}}{-2 \operatorname{Im}\left\{k_{z}\right\}} \mathrm{d} \mathbf{K} \quad .
$$

We will now introduce the only two major assumptions used throughout this work: 1) the spatial bandwidth $k_{\mathrm{BW}}$ of the electric field PWS, satisfies the condition $k_{\mathrm{BW}} \lesssim k_{0}|\chi|$, where $\chi=\sqrt{\tilde{\epsilon}}$ is the dielectric contrast of the lossy medium and 2) the medium can be described as a lossy dielectric, i.e., $\sigma \ll$ $\omega \epsilon_{0} \epsilon_{r}$. The first requirement is necessary so that the propagator modulus $|P(\mathbf{K}, z)|$ be almost flat over the entire bandwidth of the source PWS, allowing the approximation $k_{z} \simeq k_{0} \chi$; the second assumption is needed in order to simplify the final results, although closed-form expressions could be given even in a more general case. Under such conditions, (8) becomes

$$
\mathrm{TDP} \simeq \frac{\sqrt{\epsilon_{r}}}{\zeta} \int\left\|\hat{\mathbf{E}}_{\mathrm{tx}}\right\|^{2} \mathrm{~d} \mathbf{K}=\frac{\sqrt{\epsilon_{r}}}{\zeta} \mathcal{E}
$$

where $\zeta$ is the free-space wave impedance, and having defined as in signal analysis the signal energy $\mathcal{E}=\left\|\mathbf{E}_{\mathrm{tx}}(\mathbf{R})\right\|_{\mathrm{L}^{2}}^{2}$ as the square of the $L^{2}$ norm of the field distribution over $\Sigma_{0}^{+}$.

A major issue in this approach is that whenever the dielectric interface is in the near-field region of the source, coupling mechanisms will likely ensue. For a given distance $d$ between the source and the half-space, the field impinging on the dielectric interface may differ from the one that would be measured at the same distance in a free-space configuration. These phenomena notwithstanding, the approach here proposed can also be applied to near-field configurations, without any loss of generality. Indeed, the coupling between one source and a passive scatterer can be regarded as given by an infinite series of separate interactions. This can be referred to as the multiple-interaction paradigm, and it has been applied, among other scenarios, in the definition of probe-correction models for near-field measurement techniques [6].

The basic idea is that the time-domain evolution of the coupling is naturally represented through a series of simple interactions, that can be divided into scattering, transmitting and receiving events. The actual field $\hat{\mathbf{E}}_{\text {inc }}^{\|}$impinging over the half-space is thus generally not the one that would be radiated in a free-space configuration, e.g. in a far-field scenario, but rather the superposition of all the multiple-interaction contributions, i.e. the steady-state field including the actual coupling. This approach is completely general, with the farfield scenario as a special case.

\section{Sensitivity AnAlysis}

We proceed by deriving a sensitivity model for the TDP, as discussed in Section III-A.A; subsequently, this model will be used in order to investigate how the TDP sensitivity is linked to the PWS of the source (and ultimately to the field it radiates), proving in Section III-B.B that for a given configuration the sensitivity is, in general, not a deterministic value, but rather a random variable.

\section{A. Perturbation model}

The sensitivity of the TDP to variations of $\tilde{\epsilon}$ can be assessed by applying a perturbation approach to the transmission equation (6). While a perturbation of $\tilde{\epsilon}$ has a direct impact on the TDP, it will have a higher-order effect on the reflectivity of the medium interface, as seen from free-space. In other words, it will be hardly noticed by the source: it will be shown in Section VI that this idea is viable, as long as the dielectric contrast $|\chi|$ stays high. This conclusion is intuitively justified by the fact that the fundamental quantity in source-half-space 
interactions is the dielectric contrast between the two media, which is negligibly affected by the lossy medium perturbation; this phenomenon was already pointed out in [7]. Therefore, it will be assumed that the total incident electric field $\mathbf{E}_{\text {inc }}(\mathbf{R})$ fulfills the condition $\left\|\partial \mathbf{E}_{\mathrm{inc}} / \partial \tilde{\epsilon}\right\| \ll\left\|\partial \mathbf{E}_{\mathrm{tx}} / \partial \tilde{\epsilon}\right\|$.

The second order Taylor expansion of (9) with respect to the complex dielectric permittivity $\tilde{\epsilon}$ reads:

$$
\frac{\delta \mathrm{TDP}}{\mathrm{TDP}}=\frac{\delta \mathcal{E}}{\mathcal{E}}+\frac{\delta \sqrt{\epsilon_{r}}}{\sqrt{\epsilon_{r}}}+\frac{\delta^{2} \mathcal{E}}{2 \mathcal{E}}+\frac{\delta^{2} \sqrt{\epsilon_{r}}}{2 \sqrt{\epsilon_{r}}}+\frac{\delta \sqrt{\epsilon_{r}}}{\sqrt{\epsilon_{r}}} \frac{\delta \mathcal{E}}{\mathcal{E}}
$$

with:

$$
\begin{aligned}
\frac{\delta \sqrt{\epsilon_{r}}}{\sqrt{\epsilon_{r}}} & =\frac{1}{2} \frac{\delta \epsilon_{r}}{\epsilon_{r}} \\
\frac{\delta^{2} \sqrt{\epsilon_{r}}}{\sqrt{\epsilon_{r}}} & =-\frac{1}{4 \epsilon_{r}} \frac{\delta \epsilon_{r}}{\epsilon_{r}}
\end{aligned}
$$

The TDP sensitivity depends on $\delta \mathcal{E} / \mathcal{E}$ and $\delta \epsilon_{r} / \epsilon_{r}$ sensitivity terms; since the latter are trivial, we will focus our analysis on the former. Moreover, in the subsequent statistical analysis of the sensitivity, $\delta \epsilon_{r} / \epsilon_{r}$ introduces a mere offset, without accounting for the statistical dispersion of the TDP sensitivity.

We will detail here the proposed analysis method for the first-order term $\delta \mathcal{E} / \mathcal{E}$, the second-order being derived fundamentally in the same way. Knowing that the first-order differential of the norm of a complex vector $\mathbf{v}$ is related to the differential of a complex variable $p$ by

$$
\mathrm{d}\|\mathbf{v}(p)\|^{2}=2 \operatorname{Re}\left\{\mathbf{v}^{\mathrm{H}} \frac{\partial \mathbf{v}}{\partial p} \mathrm{~d} p\right\},
$$

where the apex $\mathrm{H}$ stands for the Hermitian transpose, we can write:

$$
\delta \mathcal{E}=2 \operatorname{Re}\left\{\delta \tilde{\epsilon} \int \hat{\mathbf{E}}_{\mathrm{tx}}^{\mathrm{H}} \frac{\partial \hat{\mathbf{E}}_{\mathrm{tx}}}{\partial \tilde{\epsilon}} \mathrm{d} \mathbf{K}\right\} .
$$

Using (5) and (6), together with the hypothesis of a weakly sensitive source, the following quadratic form is obtained:

$$
\delta \mathcal{E}=2 \operatorname{Re}\left\{\delta \tilde{\epsilon} \int \hat{\mathbf{E}}_{\mathrm{tx}}^{\| \mathrm{H}} \mathbf{D}_{1} \hat{\mathbf{E}}_{\mathrm{tx}}^{\|} \mathrm{d} \mathbf{K}\right\},
$$

having defined matrix $\mathbf{D}_{1}$ as follows:

$$
\begin{aligned}
& \mathbf{D}_{1}=\mathbf{T}^{\mathrm{H}} \mathbf{D}_{1}^{\prime} \\
& \mathbf{D}_{1}^{\prime}=\frac{\partial \mathbf{T}}{\partial \tilde{\epsilon}}+\mathbf{T} \frac{\partial \mathbf{\Pi}_{0}}{\partial \tilde{\epsilon}} \boldsymbol{\Pi}_{0}^{-1} .
\end{aligned}
$$

A great part of the results dealing with the sensitivity will be expressed as functions of the transmitted PWS. The rationale for this choice is that the PWS of $\mathbf{E}_{\mathrm{tx}}$ is more regular than the one in air, so that its envelope is more easily described and reproduced. This is important for the subsequent statistical analysis. Moreover, the mathematical analysis is also simplified thanks to this formalism, since the properties of the different operators are more readily applicable. In any case, the results are not affected by this choice, since the PWS $\hat{\mathbf{E}}_{\text {inc }}$ and $\hat{\mathbf{E}}_{\mathrm{tx}}$ are biunivocally related through the transmission operator.

All the derivatives involving the transmission matrices are reported in Appendix I. It is sensible to define the operator
$S: \mathbb{C}^{2 \times 2} \rightarrow \mathbb{C}$, hereafter referred to as the sensitivity operator:

$$
S(\mathbf{A})=\frac{\int \hat{\mathbf{E}}_{\mathrm{tx}}^{\| \mathrm{H}} \mathbf{A} \hat{\mathbf{E}}_{\mathrm{tx}}^{\|} \mathrm{d} \mathbf{K}}{\int\left\|\mathbf{T} \hat{\mathbf{E}}_{\mathrm{tx}}^{\|}\right\|^{2} \mathrm{~d} \mathbf{K}} .
$$

Therefore, we can now provide the following fundamental result:

$$
\frac{\delta \mathcal{E}}{\mathcal{E}}=2 \operatorname{Re}\left\{S\left(\mathbf{D}_{1}\right) \delta \tilde{\epsilon}\right\}
$$

This formulation has the advantage of pointing out the fact that the sensitivity is of course dependent on the PWS of the excitation, but that in fact its behavior is controlled by a kernel, modeled through the matrix $\mathbf{D}_{1}$, that is common to every source. In other words, $\mathbf{D}_{1}$ represents the transfer function between the PWS of the impinging wave and the sensitivity that its components will experience while being transmitted into the lossy medium.

The development applied to the linear term can also be applied to the quadratic term in (10), the only difference being that it is now necessary to consider the second-order differential of the norm of a vector $\mathbf{v}$ :

$$
\mathrm{d}^{2}\|\mathbf{v}\|^{2}=2\left\|\frac{\partial \mathbf{v}}{\partial p} \mathrm{~d} p\right\|^{2}+2 \operatorname{Re}\left\{\mathbf{v}^{\mathrm{H}} \frac{\partial^{2} \mathbf{v}}{\partial p^{2}}(\mathrm{~d} p)^{2}\right\},
$$

hence

$$
\begin{aligned}
\frac{\delta^{2} \mathcal{E}}{2 \mathcal{E}} & =\operatorname{Re}\left\{(\delta \tilde{\epsilon})^{2} S\left(\mathbf{D}_{2}\right)\right\} \\
& +|\delta \tilde{\epsilon}|^{2} S\left(\mathbf{D}_{3}\right)
\end{aligned}
$$

having introduced the following matrices:

$$
\begin{aligned}
\mathbf{D}_{2} & =\mathbf{T}^{\mathrm{H}}\left(\frac{\partial^{2} \mathbf{T}}{\partial \tilde{\epsilon}^{2}}+\mathbf{T} \frac{\partial^{2} \mathbf{\Pi}_{0}}{\partial \tilde{\epsilon}^{2}} \mathbf{\Pi}_{0}^{-1}+\right. \\
& \left.+2 \frac{\partial \mathbf{T}}{\partial \tilde{\epsilon}} \frac{\partial \boldsymbol{\Pi}_{0}}{\partial \tilde{\epsilon}} \boldsymbol{\Pi}_{0}^{-1}\right) \\
\mathbf{D}_{3} & =\mathbf{D}_{1}^{\prime \mathrm{H}} \mathbf{D}_{1}^{\prime} .
\end{aligned}
$$

The model thus derived allows computing the TDP sensitivity for a given source, just requiring as an input the PWS of the field impinging on the lossy half-space. An interesting by-product of the proposed analysis is that the frequency dependency of the TDP sensitivity is dominated by the term $\delta \tilde{\epsilon}=\delta \epsilon_{r}-\mathrm{j} \delta \sigma /\left(\omega \epsilon_{0}\right)$, as seen in (19) and (21). The fact that this term contains a $1 / f$ associated to the electrical conductivity term means that any model approximating it through a polynomial expansion is bound to need high-order terms. This is actually the case, as proved by the choice made in [4], where a third-order frequency polynomial had been necessary. The proposed representation, being derived from a physical analysis, thus leads to a more effective definition of the sensitivity.

\section{B. Statistical analysis}

The previous results can be used for investigating the following problem: how does the TDP sensitivity depend on the PWS of the source? We will here describe the PWS $\hat{\mathbf{E}}_{\mathrm{tx}}^{\|}$ as a stationary random process and study how the energy 

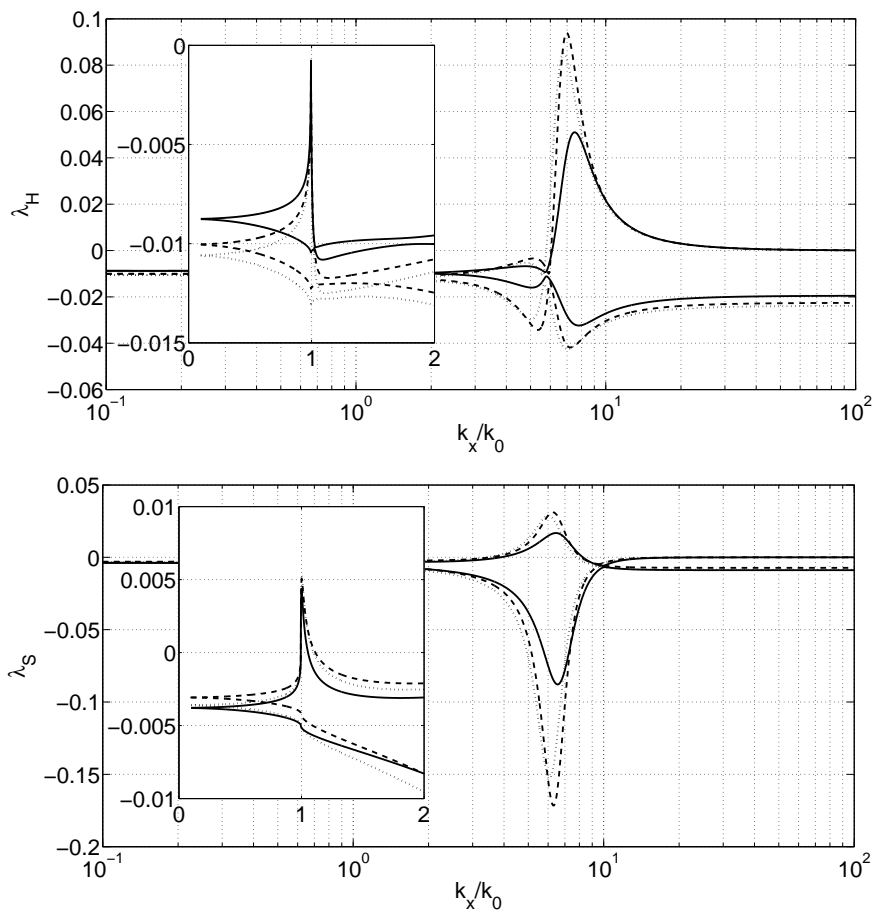

Fig. 2. Eigenvalues of the Hermitian and skew-Hermitian parts of operator $\mathbf{D}_{1}$ associated to the linear sensitivity term, at $0.9 \mathrm{GHz}$ (solid line), $2.5 \mathrm{GHz}$ (dash line) and $6 \mathrm{GHz}$ (dotted line). The results here shown regard the cut $k_{y}=0$.

sensitivity $\delta \mathcal{E} / \mathcal{E}$ is statistically distributed as a consequence of the randomness of the source PWS.

This problem can be solved easily by means of (18), since all the quantities involved can be computed directly without any need of actual simulations or measurements. Nevertheless, (18) requires to impose a certain PWS distribution; it is possible to prove that the sensitivity actually depends just on the envelope of the PWS, since (18) involves quadratic expressions [8] (see Appendix II). For this reason, the energynormalized spectrum envelope $w(\mathbf{K})$ will be considered, as defined by:

$$
w(\mathbf{K})=\frac{\left\|\hat{\mathbf{E}}_{\mathrm{tx}}^{\|}\right\|^{2}}{\int\left\|\hat{\mathbf{E}}_{\mathrm{tx}}^{\|}\right\|^{2} \mathrm{~d} \mathbf{K}} .
$$

In the context of a statistical analysis, the sensitivity operator defined in (18) can be evaluated directly; the focus will be put on studying the first two statistical moments of the sensitivity for a given PWS energy-density spectrum $\langle w(\mathbf{K})\rangle$. Since all the quantities in (19) and (21) are deterministic but for the sensitivity operator, the statistical analysis will focus on this last term.

In particular, the following approximation holds (see Appendix II) as long as the spatial bandwidth $k_{\mathrm{BW}}$ of the normalized energy-density spectrum $\langle w(\mathbf{K})\rangle$ of the average source is smaller than $k_{0}|\chi|$ :

$$
\langle S(\mathbf{A})\rangle \simeq \int\left[\bar{\lambda}_{H}(\mathbf{K})+\bar{\lambda}_{S}(\mathbf{K})\right]\langle w(\mathbf{K})\rangle \mathrm{d} \mathbf{K} \quad,
$$

where $\bar{\lambda}_{H}$ and $\bar{\lambda}_{S}$ are the arithmetic means of, respectively, the eigenvalues of the Hermitian and the skew-Hermitian parts
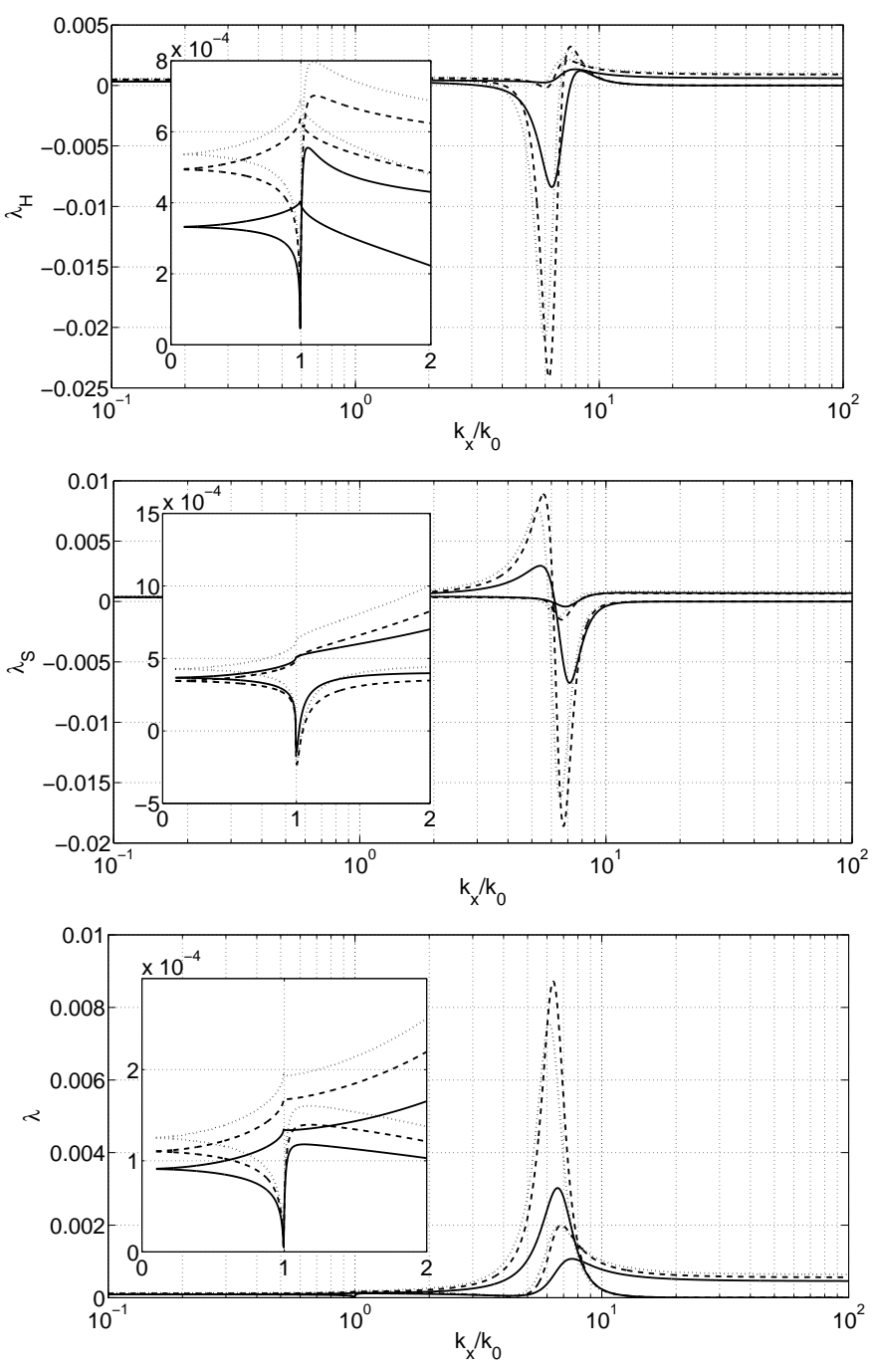

Fig. 3. Eigenvalues of the Hermitian and skew-Hermitian parts of operator $\mathbf{D}_{2}$ and $\mathbf{D}_{3}$ (last picture) associated to the quadratic sensitivity term, at $0.9 \mathrm{GHz}$ (solid line), $2.5 \mathrm{GHz}$ (dash line) and $6 \mathrm{GHz}$ (dotted line). The results here shown regard the cut $k_{y}=0$.

of matrix $\mathbf{A}$, where this last stands for any of the derivative operators $\mathbf{D}_{i}$. This result is noteworthy, since it states that the average of the sensitivity over all the possible sources depends exclusively on the properties of the lossy medium and the average spectral content of the source.

The dispersion of the TDP sensitivity around its average can be assessed by studying the eigenvalues of the three derivative operators $\mathbf{D}_{i}$. Indeed:

$$
\begin{aligned}
\int \min \lambda_{H} w \mathrm{~d} \mathbf{K} \leq S\left(\mathbf{A}_{H}\right) & \leq \int \max \lambda_{H} w \mathrm{~d} \mathbf{K} \\
\int \min \lambda_{S} w \mathrm{~d} \mathbf{K} \leq S\left(\mathbf{A}_{S}\right) & \leq \int \max \lambda_{S} w \mathrm{~d} \mathbf{K}
\end{aligned}
$$

so that, the distance between the eigenvalue pairs is a direct measure of the dispersive nature of the sensitivity.

In order to test these ideas, we will hereafter consider a planar SAR assessment configuration as a practical application, since it is an interesting example of high-contrast lossy configuration; the nominal electrical parameters of the lossy 


\begin{tabular}{cccc}
\hline \hline Freq. (GHz) & $\epsilon_{r}$ & $\sigma(\mathrm{S} / \mathrm{m})$ & $|\chi|$ \\
\hline 0.9 & 41.5 & 0.97 & 6.77 \\
2.5 & 39.2 & 1.8 & 6.42 \\
6 & 36.2 & 4.45 & 6.23 \\
\hline \hline
\end{tabular}

TABLE I

THE ELECTRICAL CHARACTERISTICS OF TISSUE-EQUIVALENT LIQUIDS FOR SAR ASSESSMENT CONFIGURATIONS, AS SET IN [9].

medium are thus imposed by international standards, such as [9], and are reported in Table I.

Therefore, the associated eigenvalues are shown in Fig. 2 and 3 , as computed for the three half-space configurations of Table I. The spectral variable was normalized with respect to the free-space wave-number $k_{0}$. Matrices $\mathbf{D}_{1}$ and $\mathbf{D}_{2}$ were expanded into their Hermitian and skew-Hermitian components, as explained in Appendix II, whereas $\mathbf{D}_{3}$ is already Hermitian.

Figures 2 and 3 show that each pair of eigenvalues coincides around the origin $\mathbf{K}=\mathbf{0}$, i.e., for normal paraxial incidence. The $L^{2}$-integrable discontinuities around $k_{0}$ are due to the branch singularity in the spectrum of Green's function [10]; a similar behavior is present around $k_{0}|\chi|$, fundamentally due to the same phenomena, but mitigated by losses. Conversely, it can be noticed that the distance between each pair of eigenvalues widens with the time and space frequency. This trend gets much stronger when getting beyond $k_{0}$, i.e., when considering reactive components of the source PWS. More specifically, the skew-Hermitian components present an increased distance with respect to the purely Hermitian ones for a given K. Looking at (19), the skew-Hermitian component operates over the variations in the conductivity $\sigma$ : this means that the reactive components of the source PWS will be affected in a more variable way in response to variations of the conductivity than equal-strength modifications of the permittivity. These simple conclusions already show that the sensitivity will present an higher degree of variability for nearfield sources, richer in reactive energy, than far-field ones, where no reactive component is available; this is coherent with the common-sense perception that the TDP sensitivity is not dispersed for a far-field configuration.

In order to assess these findings, the sensitivity operators were studied in several ways: 1) equation (25) was used for assessing the average sensitivity, 2) a deterministic estimate was given assuming a paraxial propagation, thus considering the approximation $S(\mathbf{D})(\mathbf{K}) \simeq S(\mathbf{D})(\mathbf{0})$; and finally 3 ) equation (18) was directly evaluated by considering a population of ten thousand random realizations for the transmitted PWS. For this last case, we considered a Gaussian energy-density spectrum with a $-3 \mathrm{~dB}$ bandwidth $k_{\mathrm{BW}}=k_{0}$, with a zero-mean Gaussian distribution. This choice is justified in the context of SAR assessment tests for telecommunications, where sources are usually not highly directive. Table II shows the results of this analysis. It turns out that the paraxial approximation is not very effective when compared to (25); nevertheless, it allows to introduce a very simple model for the average sensitivity, as

\begin{tabular}{lcrrrrr}
\hline \hline & & $\mathbf{D}_{1 H}$ & $\mathrm{j} \mathbf{D}_{1 S}$ & $\mathbf{D}_{2 H}$ & $\mathrm{jD}_{2 S}$ & $\mathbf{D}_{3}$ \\
\hline \multirow{5}{*}{$0.9 \mathrm{GHz}$} & $\langle S\rangle$ & -94 & 38 & 3.6 & -3.9 & 1.0 \\
& eq. $(25)$ & -94 & 41 & 3.6 & -4.1 & 1.1 \\
& paraxial & -88 & 38 & 3.3 & -3.7 & 0.91 \\
& $\left\langle S^{2}\right\rangle$ & 9.2 & 3.8 & 0.36 & 0.38 & 0.10 \\
\hline \multirow{2}{*}{$2.5 \mathrm{GHz}$} & $\langle S\rangle$ & -113 & 36 & 5.7 & -4.2 & 1.4 \\
& eq. $(25)$ & -108 & 34 & 5.5 & -4.0 & 1.3 \\
& paraxial & -100 & 31 & 4.9 & -3.4 & 1.1 \\
& $\left\langle S^{2}\right\rangle$ & 11.0 & 3.4 & 0.53 & 0.39 & 0.13 \\
\hline \multirow{2}{*}{$6.0 \mathrm{GHz}$} & $\langle S\rangle$ & -113 & 39 & -5.8 & 4.8 & 1.5 \\
& eq. $(25)$ & -114 & 40 & -5.9 & 4.9 & 1.5 \\
& paraxial & -106 & 36 & -5.4 & 4.3 & 1.3 \\
& $\left\langle S^{2}\right\rangle$ & 11 & 3.9 & 0.58 & 0.47 & 0.14 \\
\hline \hline
\end{tabular}

TABLE II

SENSITIVITY OPERATORS FOR A GAUSSIAN-ENVELOPE PWS, WITH BANDWIDTH $k_{0}$. ALL THE RESULTS MUST BE MULTIPLIED BY A FACTOR $10^{-4}$.

reported in Section VI. Much worse is the fact that the paraxial approximation fails to acknowledge the intrinsic variability of the sensitivity with respect to the source PWS; the sensitivity is indeed collapsed into a deterministic value, rather than a statistical distribution.

Statistical distributions for the five operators are shown in Fig. 4 for the same Gaussian PWS envelope, but for a changing spectral bandwidth $k_{\mathrm{BW}}$, for a frequency of $6 \mathrm{GHz}$. It appears that the case $k_{\mathrm{BW}}=k_{0} / 2$, i.e., with very little reactive components, is indeed well described by the paraxial approximation; but as soon as the reactive content increases, the sensitivity moves away and spreads. This trend is in accordance with the results presented in Fig. 2 and 3 and the previous considerations about the distance between the eigenvalues. It is therefore not possible to neglect the variability of the sensitivity due to the source characteristics. The spread is quantified in Table II for $k_{\mathrm{BW}}=k_{0}$, showing that considering a $95 \%$ margin of confidence implies an uncertainty of $\pm 20 \%$ for the sensitivity, approximating the distributions as Gaussian ones.

\section{INCLUDING THE PRESENCE OF BUFFER LAYERS}

In many practical cases the lossy dielectric may not face directly the air half-space, but buffer layers of complex dielectric permittivity $\tilde{\epsilon}_{s i}$ and thickness $s_{i}$ are interposed between the two half-spaces, for $i \in\left[1, N_{b}\right]$ where $N_{b}$ is the number of buffer layers. Although the previous model was developed under a half-space assumption, it can be promptly extended to a stratified configuration; in the following discussion, we will assume that all the media have negligible losses with respect to the inner medium. Therefore, for the sake of computing the TDP, the only important quantity is the PWS $\hat{\mathbf{E}}_{\mathrm{tx}}^{\|}$of the field transmitted into the lossy dielectric. This can be computed directly in the case of a stratified structure, by cascading the relationships reported in Appendix I. In order to simplify the notations, hereafter all of the quantities related to the electric field will refer to the tangential component. 

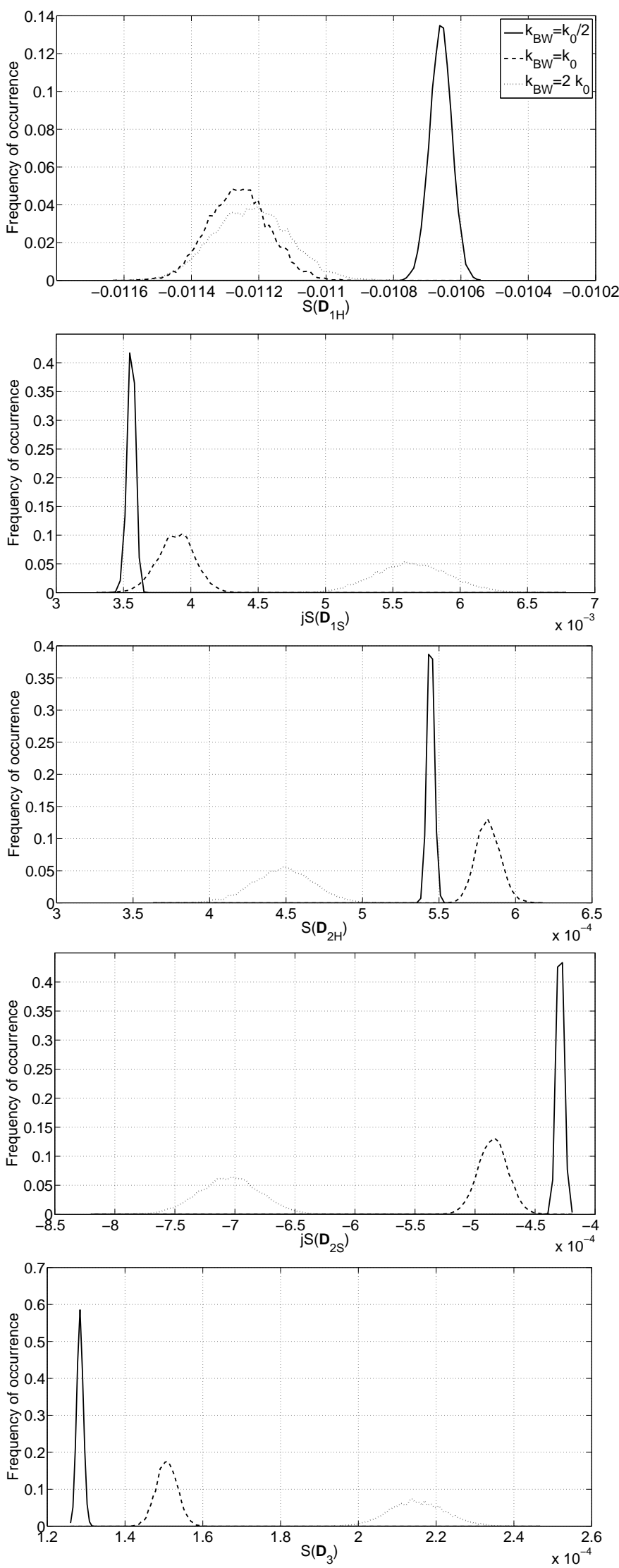

Fig. 4. Statistical distributions of the sensitivity operators for the Hermitian and skew-Hermitian components of the three derivative matrices $\mathbf{D}_{i}$, as computed for a Gaussian-distributed PWS with a Gaussian-envelope energydensity function with $-3 \mathrm{~dB}$ spatial bandwidth $k_{\mathrm{BW}} / k_{0}$ equal to $0.5,1$ and 2. The working frequency is $6 \mathrm{GHz}$ and the lossy medium is specified in Table I.

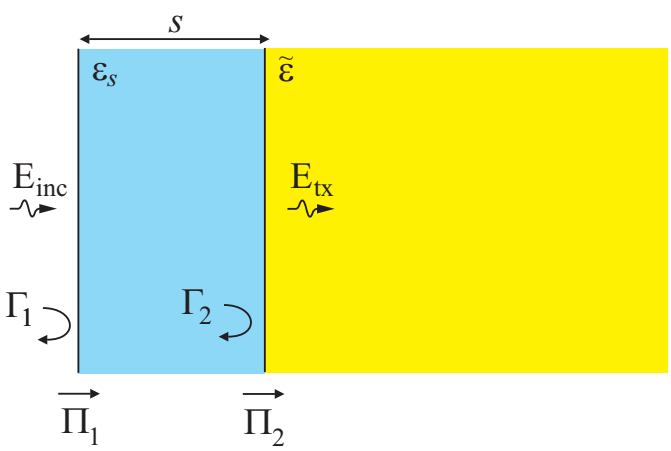

Fig. 5. A stratified configuration with a lossless buffer layer of thickness $s$.

In this Section we first investigate how the presence of a buffer layer affects the TDP, in particular by extending the analysis of the role of the reactive energy of the source. Then, we focus on the modification of the TDP sensitivity.

\section{A. Impact on the TDP}

The PWS $\hat{\mathbf{E}}_{\mathrm{tx}}$ of the field transmitted into the lossy medium can be regarded as having been generated in a configuration involving just two media, i.e., the right half-space made of the lossy dielectric and the left one in air, as in the original description. To this end, an equivalent source needs to be introduced, much in the same way as for a Thevenin equivalent circuit. For the case of a single additional layer as depicted in Fig. 5, we can write:

$$
\hat{\mathbf{E}}_{\mathrm{tx}}=\boldsymbol{\Pi}_{2} \mathbf{R}^{-1} \boldsymbol{\Pi}_{1} \mathrm{e}^{-\mathrm{j} k_{z s} s} \hat{\mathbf{E}}_{\mathrm{inc}},
$$

with

$$
\mathbf{R}=\mathbf{1}+\mathrm{e}^{-2 \mathrm{j} k_{z s} s} \boldsymbol{\Gamma}_{1} \boldsymbol{\Gamma}_{2}
$$

Matrices $\boldsymbol{\Gamma}_{1}$ and $\boldsymbol{\Gamma}_{2}$ are the reflection matrices at the two dielectric interfaces, as given in Appendix I, whereas $k_{z 0}$ and $k_{z s}$ are the longitudinal propagation constants for, respectively, the free-space and the shell media.

The PWS transmitted in the non-stratified configuration was given by (6):

$$
\hat{\mathbf{E}}_{\mathrm{tx}}=\boldsymbol{\Pi}_{0} \hat{\mathbf{E}}_{\mathrm{inc}} \mathrm{e}^{-\mathrm{j} k_{z 0} s} .
$$

By comparing (28) and (30), it is possible to define an equivalent PWS $\hat{\mathbf{E}}_{\text {eq }}$ impinging from the air side (with no shell) as :

$$
\hat{\mathbf{E}}_{\mathrm{tx}}=\boldsymbol{\Pi}_{0} \hat{\mathbf{E}}_{\mathrm{eq}} \mathrm{e}^{-\mathrm{j} k_{z 0} s}=\boldsymbol{\Pi}_{0} \boldsymbol{\Xi} \hat{\mathbf{E}}_{\mathrm{inc}} \mathrm{e}^{-\mathrm{j} k_{z 0} s},
$$

having introduced the correction matrix $\Xi$

$$
\boldsymbol{\Xi}=\boldsymbol{\Pi}_{0}^{-1} \boldsymbol{\Pi}_{2} \mathbf{R}^{-1} \boldsymbol{\Pi}_{1} \mathrm{e}^{-\mathrm{j}\left(k_{z 2}-k_{z 0}\right) s} .
$$

Under a weakly-sensitive source assumption, the impact of the shell on the TDP can be assessed by computing the ratio of the signal energy $\mathcal{E}_{s}$ for the shell configuration and the original one $\mathcal{E}_{0}$, that are related to the definition of the TDP given in (9), while expressing all the spectral quantities as functions of the transmitted PWS:

$$
\frac{\mathrm{TDP}_{s}}{\mathrm{TDP}_{0}}=\frac{\mathcal{E}_{s}}{\mathcal{E}_{0}}=\frac{\left\|\boldsymbol{\Pi}_{0} \boldsymbol{\Xi} \boldsymbol{\Pi}_{0}^{-1} \mathbf{E}_{\mathrm{tx}, 0}\right\|_{L^{2}}^{2}}{\left\|\mathbf{E}_{\mathrm{tx}, 0}\right\|_{L^{2}}^{2}},
$$




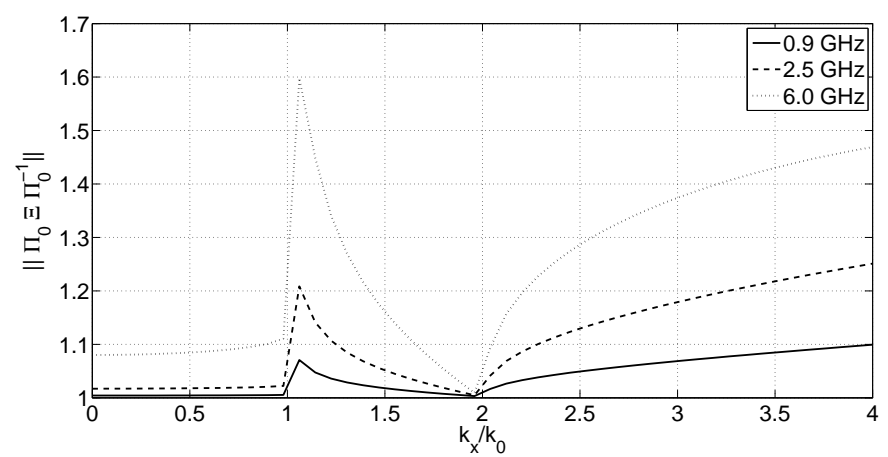

Fig. 6. The norm of matrix $\boldsymbol{\Xi}$ for a $2 \mathrm{~mm}$ thick buffer with $\epsilon_{s}=4$. The inner lossy dielectric are tissue-equivalent liquids as described in Table I.

where $\mathbf{E}_{\mathrm{tx}, 0}$ is the PWS transmitted with no shell present. The previous expression can be bound as

$$
\frac{\mathcal{E}_{s}}{\mathcal{E}_{0}} \leq \int\left\|\boldsymbol{\Pi}_{0} \boldsymbol{\Xi} \boldsymbol{\Pi}_{0}^{-1}\right\|^{2} w(\mathbf{K}) \mathrm{d} \mathbf{K}
$$

This formulation can be usefully employed in assessing the overall effect of a buffer layer on the TDP. Indeed, the matrix $\boldsymbol{\Pi}_{0} \boldsymbol{\Xi} \boldsymbol{\Pi}_{0}^{-1}$ accounts for the modification of the transmissivity of the PWS from the air side to the lossy medium in the presence of a buffer layer; again, its effect is weighted by the energy-normalized spectrum $w(\mathbf{K})$.

The norm of $\boldsymbol{\Xi}$ is related to

$$
\|\boldsymbol{\Xi}\| \leq\left\|\boldsymbol{\Pi}_{1}\right\|\left\|\boldsymbol{\Pi}_{2}\right\|\left\|\mathbf{R}^{-1}\right\| \quad ;
$$

the first two norms are bound to have a finite value, due to their physical meaning: they account for the transmissivity. On the other hand, $\left\|\mathbf{R}^{-1}\right\|$ may be unbounded, since it represents standing-wave phenomena. This can lead to a strong increase in the TDP as soon as a resonance can be physically instated inside the shell region.

An example of the behavior of $\left\|\boldsymbol{\Pi}_{0} \boldsymbol{\Xi} \boldsymbol{\Pi}_{0}^{-1}\right\|$ is given in Fig. 6, for the case of SAR tissue-equivalent liquids with a shell $2 \mathrm{~mm}$ thick, with a relative permittivity $\epsilon_{s}=4$. These results clearly prove that the TDP is indeed modified by the presence of the buffer, especially (as expected) at those frequencies where its thickness is comparable with the wavelength. These conclusions support and complete the findings reported in [12], [13].

Fig. 6 shows that the shell affects the TDP in two ways: 1) in the visible region (with respect to air), the shell operates as an impedance transformer, providing a better matching between the wave-impedances in air and in the lossy dielectric; 2) in the reactive region, it allows the transmission of more reactive energy $\left(\|\mathbf{K}\|>k_{0}\right)$ if $1<\epsilon_{s}<\epsilon_{r}$, since it behaves as a lens focusing PWS components towards the direction normal to the dielectric interfaces. It is this last phenomenon that gives the strongest contribution to the TDP modification. As a consequence, modification of the reactive part may have a non negligible effect on the topography of the electric field inside the lossy medium.

As in the case treated in Section III-B.B, the impact of a shell on the TDP depends on the reactive content of the

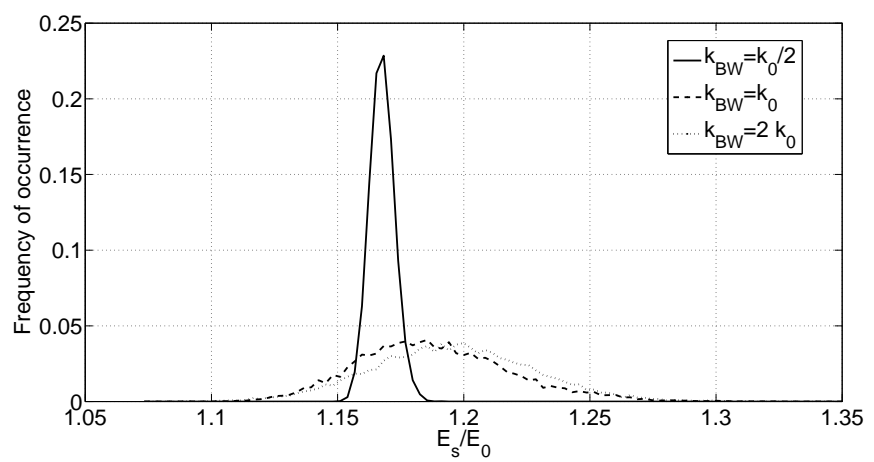

Fig. 7. Statistical distributions of the modification factor $\mathcal{E}_{s} / \mathcal{E}_{0}$ for the TDP, due to the presence of a dielectric shell for a $6 \mathrm{GHz}$ SAR configuration.

impinging PWS. This same approach yields the results shown in Fig. 7 : although the average modification of the TDP is not strongly affected, the more reactive the source, the more statistically dispersed is the TDP. It is fundamental to bear in mind that the scenario here considered presents a perfect knowledge of the electrical characteristics of the lossy medium.

The effectiveness of the bound given in (34) is proven in Table III, where for the $6 \mathrm{GHz}$ configuration, with $k_{B W}=$ $k_{0}$, the maximum modification of 1.41 well represents the dispersiveness shown in Figure 7.

\section{B. Impact on the TDP sensitivity}

The equivalent-source approach can now be applied to derive the sensitivity of the TDP in the stratified configuration, yielding

$$
\begin{aligned}
\frac{\partial \hat{\mathbf{E}}_{\mathrm{tx}}}{\partial \tilde{\epsilon}} & =\frac{\partial \boldsymbol{\Pi}_{2}}{\partial \tilde{\epsilon}} \mathbf{R}^{-1} \boldsymbol{\Pi}_{1} \mathrm{e}^{-\mathrm{j} k_{z s} s} \hat{\mathbf{E}}_{\mathrm{inc}}+ \\
& -\boldsymbol{\Pi}_{2} \mathrm{e}^{-\mathrm{j} k_{z s} s} \mathbf{R}^{-1} \boldsymbol{\Gamma}_{1} \frac{\partial \boldsymbol{\Gamma}_{2}}{\partial \tilde{\epsilon}} \mathbf{R}^{-1} \mathrm{e}^{-2 \mathrm{j} k_{z s} s} \boldsymbol{\Pi}_{1} \hat{\mathbf{E}}_{\mathrm{inc}}
\end{aligned}
$$

As shown in Section VI, the sensitivity of the reflection matrices is one order of magnitude smaller than that of the transmission ones. Hence, we can claim that:

$$
\frac{\partial \hat{\mathbf{E}}_{\mathrm{tx}}}{\partial \tilde{\epsilon}} \simeq \frac{\partial \boldsymbol{\Pi}_{2}}{\partial \tilde{\epsilon}} \mathbf{R}^{-1} \boldsymbol{\Pi}_{1} \mathrm{e}^{-\mathrm{j} k_{z s} s} \hat{\mathbf{E}}_{\mathrm{inc}} .
$$

Comparing this result to the sensitivity obtained in the first place with no buffer yields:

$$
\frac{\partial \hat{\mathbf{E}}_{\mathrm{tx}}}{\partial \tilde{\epsilon}} \simeq \frac{\partial \boldsymbol{\Pi}_{0}}{\partial \tilde{\epsilon}} \boldsymbol{\Xi}^{\prime} \hat{\mathbf{E}}_{\mathrm{inc}} \mathrm{e}^{-\mathrm{j} k_{z s} s},
$$

where

$$
\boldsymbol{\Xi}^{\prime}=\left(\frac{\partial \boldsymbol{\Pi}_{0}}{\partial \tilde{\epsilon}}\right)^{-1} \frac{\partial \boldsymbol{\Pi}_{2}}{\partial \tilde{\epsilon}} \mathbf{R}^{-1} \boldsymbol{\Pi}_{1} \mathrm{e}^{-\mathrm{j}\left(k_{z 2}-k_{z 0}\right) s} .
$$

Plugging (31) and (38) into (14) provides a tool for computing the new linear sensitivities as in the case with no shell. The fundamental quantity that dominates the modification of the sensitivities is the product of the norms of the two matrices $\boldsymbol{\Xi}$ and $\boldsymbol{\Xi}^{\prime}$. An example of the spectral behaviour of $\left\|\boldsymbol{\Xi}^{\prime}\right\|$ is shown in Fig. 8: as opposed to the modification of the TDP, the 


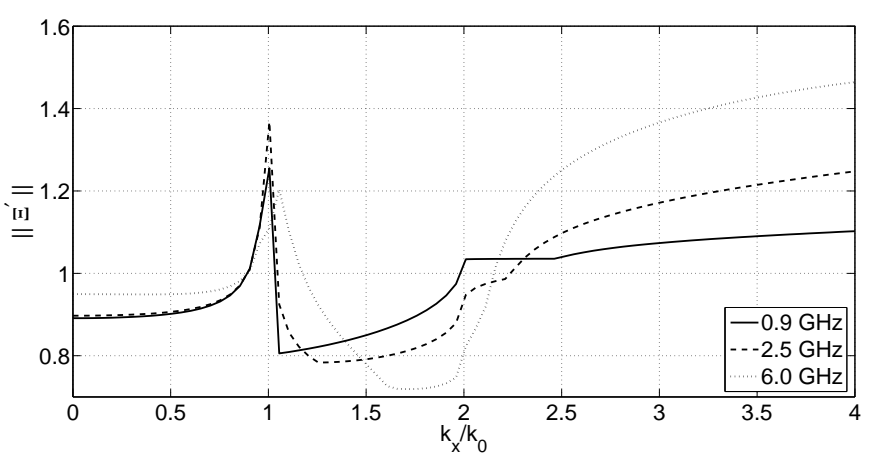

Fig. 8. The norm of matrix $\boldsymbol{\Xi}^{\prime}$ for a $2 \mathrm{~mm}$ thick buffer with $\epsilon_{s}=4$.

norm of the first derivative of the transmitted PWS is generally not strongly affected when compared to the case with no shell, especially for sources with a poor reactive content. Again, the statistical distributions related to the source PWS have been computed and are shown in Fig. 9. These results give a better insight into the modification of the linear sensitivities; indeed, the sensitivity to the conductivity (imaginary part of $S$ ) is more strongly affected than the one to the permittivity. In particular, its average value decreases for a more reactive source and it spreads over a quite larger support.

The derivation of a closed-form upper-bound is not feasible for the modification of the linear sensitivities. Nevertheless, the following bound provides some information:

$$
\begin{gathered}
\frac{\left|S\left(\mathbf{D}_{1 H}\right)+\mathrm{j} S\left(\mathbf{D}_{1 S}\right)\right|_{s}}{\left|S\left(\mathbf{D}_{1 H}\right)+\mathrm{j} S\left(\mathbf{D}_{1 S}\right)\right|_{0}} \leq \\
\leq \int\left\|\boldsymbol{\Pi}_{0} \boldsymbol{\Xi} \boldsymbol{\Pi}_{0}^{-1}\right\|^{2}\left\|\boldsymbol{\Xi}^{\prime}\right\|^{2} w(\mathbf{K}) \mathrm{d} \mathbf{K},
\end{gathered}
$$

where the indices stands for "shell" and "no shell".

The statistical results for the modification of the TDP and its sensitivities are summarized in Table III for $k_{B W}=k_{0}$, together with the value of the correction factors evaluated for a paraxial propagation and the upper bounds given in (34) and (40). As for the results shown in Table II, though the paraxial model provides a fairly good estimate for the average modifications, it is unable to account for the dispersion they induce. Moreover, it cannot explain neither the stronger impact for more reactive sources, nor the shift in the linear sensitivity related to the conductivity (see Fig. 9).

For the sake of brevity, we will not derive here the secondorder sensitivity in the case of a shell. Nevertheless, the same approach can be extended to include such analysis. We can conclude that, in the case of SAR applications, the presence of a shell has not a fundamental impact on how the TDP reacts to modifications in the permittivity $\epsilon_{r}$, whereas the sensitivity to the conductivity $\sigma$ is more strongly spread and lowered. Moreover, the shell can indeed strongly increase the transfer of energy between the two half-spaces, as well as lead to a further uncertainty in the evaluation of the TDP for a given near-field source.
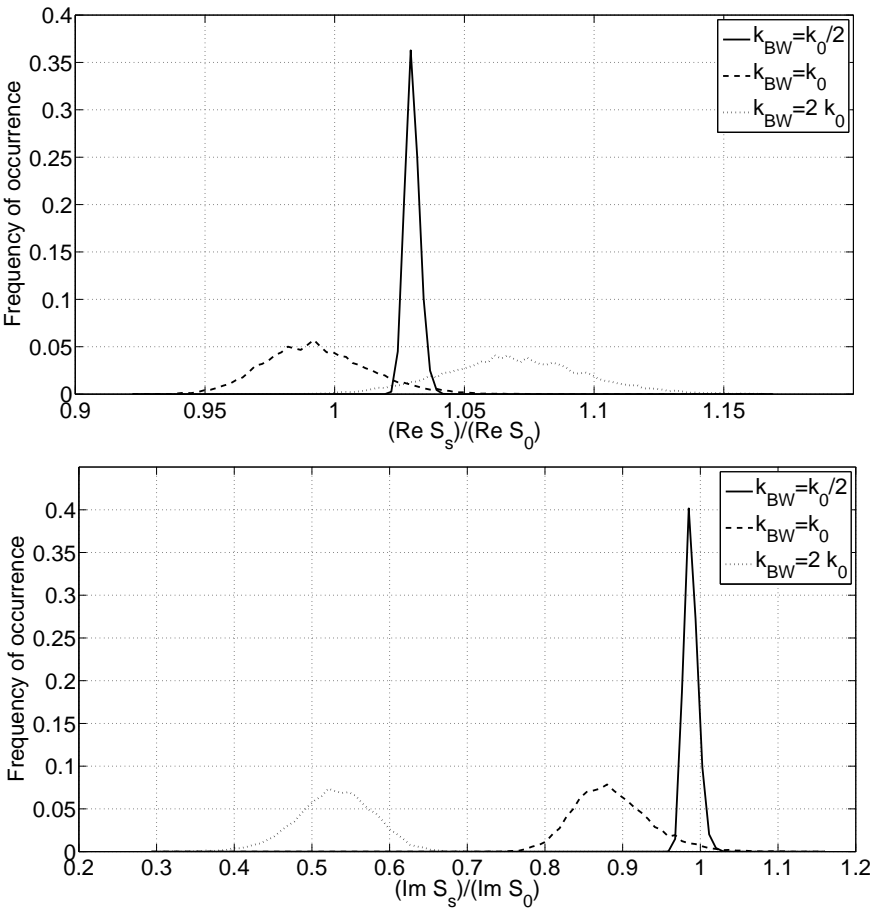

Fig. 9. Statistical distributions of the modification of the linear sensitivity operator due to the presence of a dielectric shell for a $6 \mathrm{GHz}$ SAR configuration. The real part is related to the sensitivity to the dielectric permittivity

\begin{tabular}{|c|c|c|c|c|}
\hline & & TDP & $S\left(\mathbf{D}_{1 H}\right)$ & $S\left(\mathbf{D}_{1 S}\right)$ \\
\hline $0.9 \mathrm{GHz}$ & $\begin{array}{c}\langle\cdot\rangle \\
\langle\cdot 2\rangle \\
\text { paraxial } \\
\text { upper bound }\end{array}$ & $\begin{array}{c}1.008 \\
0.0034 \\
1.01 \\
1.009\end{array}$ & $\begin{array}{c}0.87 \\
0.0066 \\
0.92 \\
(1.07)\end{array}$ & $\begin{array}{c}0.80 \\
0.02 \\
0.86 \\
-\end{array}$ \\
\hline $2.5 \mathrm{GHz}$ & $\begin{array}{c}\langle\cdot\rangle \\
\langle\cdot 2\rangle \\
\text { paraxial } \\
\text { upper bound }\end{array}$ & $\begin{array}{c}1.04 \\
0.010 \\
1.03 \\
1.03\end{array}$ & $\begin{array}{c}0.88 \\
0.0074 \\
0.92 \\
(1.13)\end{array}$ & $\begin{array}{c}0.79 \\
0.034 \\
0.88 \\
-\end{array}$ \\
\hline $6.0 \mathrm{GHz}$ & $\begin{array}{c}\langle\cdot\rangle \\
\left\langle\cdot{ }^{2}\right\rangle \\
\text { paraxial } \\
\text { upper bound }\end{array}$ & $\begin{array}{l}1.19 \\
0.03 \\
1.16 \\
1.41\end{array}$ & $\begin{array}{c}0.99 \\
0.02 \\
1.02 \\
(1.33)\end{array}$ & $\begin{array}{l}0.89 \\
0.05 \\
0.98 \\
-\end{array}$ \\
\hline
\end{tabular}
$\epsilon_{r}$, while the imaginary part deals with the sensitivity to the conductivity $\sigma$.

TABLE III

MODIFICATION OF THE TDP AND ITS LINEAR SENSITIVITIES IN PRESENCE OF A DIELECTRIC SHELL, WITH $s=2 \mathrm{MM}$ AND $\epsilon_{s}=4$, FOR A

GAUSSIAN-ENVELOPE PWS WITH $k_{\mathrm{BW}}=k_{0}$. THE BOUND FOR THE TDP MODIFICATION IS GIVEN BY (34), WHEREAS THE VALUES IN PARENTHESES CORRESPOND TO THE BOUND (40). 


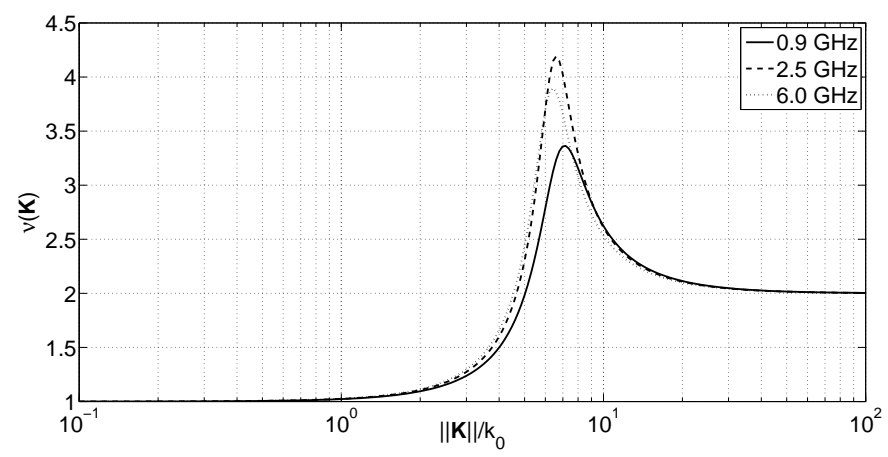

Fig. 10. The maximum eigenvalue of the matrix $\mathbf{T}^{\mathrm{H}} \mathbf{T}$, as computed for the three SAR configurations in Table I.

\section{NEGLECTING THE NORMAL COMPONENT}

There have been several discussions about whether the normal component $\hat{E}_{z}$ be negligible or not [3]. In other words, is it possible to approximate $\|\hat{\mathbf{E}}\|$ with $\left\|\hat{\mathbf{E}}^{\|}\right\|$? This same assumption is used throughout this paper. An answer to this question can be given by recalling that [11]

$$
\|\hat{\mathbf{E}}\|^{2}=\left\|\mathbf{T} \hat{\mathbf{E}}^{\|}\right\|^{2}=\hat{\mathbf{E}}^{\| \mathrm{H}} \mathbf{T}^{\mathrm{H}} \mathbf{T} \hat{\mathbf{E}}^{\|} \leq \nu(\mathbf{K})\left\|\hat{\mathbf{E}}^{\|}\right\|^{2},
$$

where $\nu(\mathbf{K})$ is the maximum eigenvalue of the matrix $\mathbf{T}^{\mathrm{H}} \mathbf{T}$. This allows writing

$$
1 \leq \frac{\|\hat{\mathbf{E}}(\mathbf{K})\|^{2}}{\left\|\hat{\mathbf{E}}^{\|}(\mathbf{K})\right\|^{2}} \leq \nu(\mathbf{K})
$$

A useful rule of thumb for deciding whether the normal component can be neglected involves again the hypothesis $k_{\mathrm{BW}} \lesssim k_{0}|\chi|$ : in this case, the normal component can be neglected. As an example validating this statement, the upper bound $\nu(\mathbf{K})$ is shown in Fig. 10 for several frequencies, as computed for standard tissue-equivalent liquids.

Back in the spatial domain, the impact of $E_{z}$ on the TDP can also be assessed; indeed:

$$
1 \leq \frac{\mathrm{TDP}}{\mathrm{TDP}^{\|}} \leq \int \nu(\mathbf{K}) w(\mathbf{K}) \mathrm{d} \mathbf{K}
$$

having defined TDP ${ }^{\|}$as the TDP obtained by neglecting $E_{z}$. The band-limitedness of the PWS means that the upper-bound for the error on the TDP for SAR configurations must be lower than $4 \%$ for a Gaussian-envelope PWS with $k_{\mathrm{BW}}=k_{0}$ and $1 \%$ for $k_{\mathrm{BW}}=k_{0} / 2$, whereas it can be as high as $20 \%$ for $k_{\mathrm{BW}}=2 k_{0}$.

\section{PARAXIAL APPROXIMATION}

In the previous analysis, no assumption was made on the distance between the source and the dielectric interface, so that the reactive components (in air) of the PWS were considered as contributing to the TDP. Nevertheless, the propagator $P(\mathbf{K}, z)$ in air imposes a very strong attenuation to the reactive parts, as expressed by the attenuation-per-wavelength $A_{\mathrm{dB} / \lambda}$ :

$$
A_{\mathrm{dB} / \lambda}\left(\mathbf{K}, \frac{z}{\lambda}\right)=40 \pi \log (\mathrm{e}) \frac{z}{\lambda} \sqrt{\left(\frac{\|\mathbf{K}\|}{k_{0}}\right)^{2}-1} .
$$

As an example, the PWS components over the spectral radius $\|\mathbf{K}\|=1.1 k_{0}$ experience an attenuation of $25 \mathrm{~dB}$ after a propagation distance of one wavelength. In many practical situations such a distance is not enough to justify a far-field analysis; nevertheless, although the field topography has not yet assumed its far-field configuration, a distance of $\lambda$ is enough to enforce a PWS with almost the same envelope $w(\mathbf{K})$ that would be obtained in a truly far-field analysis. Thus, from the TDP point of view, these two configurations coincide. Recalling that the eigenvalues of all the sensitivity operators vary very slowly over the air active region (see Fig. 2 and 3), they can be fairly approximated by considering their values in the origin. This means that even in a configuration that does not satisfy the far-field criteria, a paraxial analysis can be applied.

Under a paraxial approach, the transmission operator $\boldsymbol{\Pi}_{0}$ is just a scalar function, given by

$$
\Pi_{0}=\frac{2}{1+\chi},
$$

to be compared with the exact expression (55). It is thus necessary to define the sensitivities of the transmission terms:

$$
\begin{gathered}
\frac{1}{\Pi_{0}} \frac{\partial \Pi_{0}}{\partial \tilde{\epsilon}} \simeq-\frac{1}{2 \chi(1+\chi)} \\
\frac{1}{\Pi_{0}} \frac{\partial^{2} \Pi_{0}}{\partial \tilde{\epsilon}^{2}} \simeq \frac{1+3 \chi}{4 \chi^{3}(1+\chi)^{2}},
\end{gathered}
$$

and, finally, the sensitivities of the signal energy $\mathcal{E}$ as required by (10)

$$
\begin{aligned}
\frac{\delta \mathcal{E}}{\mathcal{E}} & \simeq-\operatorname{Re}\left\{\frac{\delta \tilde{\epsilon}}{\chi(1+\chi)}\right\} \\
\frac{\delta^{2} \mathcal{E}}{\mathcal{E}} & \simeq \frac{1}{2}\left|\frac{\delta \tilde{\epsilon}}{\chi(1+\chi)}\right|^{2}+ \\
& +\frac{1}{2} \operatorname{Re}\left\{\frac{1+3 \chi}{\chi^{3}(1+\chi)^{2}}(\delta \tilde{\epsilon})^{2}\right\} .
\end{aligned}
$$

These results can be applied in practice as long as the reactive content of a source is negligible, thus providing a simple way of predicting the sensitivity of the TDP together with (10).

It is also interesting to consider the reflection coefficient $\Gamma_{d}$ at the air-dielectric interface as seen from air and its relative sensitivity:

$$
\begin{aligned}
\Gamma_{d} & =\frac{\chi-1}{\chi+1} \\
\left|\frac{1}{\Gamma_{d}} \frac{\partial \Gamma_{d}}{\partial \tilde{\epsilon}}\right| & \simeq \frac{1}{\left|\chi\left(\chi^{2}-1\right)\right|} .
\end{aligned}
$$

This last result can be used to explain the low sensitivity of the incident field to drifts in the lossy medium. To this end, let us consider the power $P_{R}=P_{\mathrm{av}}\left(1-\left|\Gamma_{\text {in }}\right|^{2}\right)$ radiated by a lossless antenna; $\Gamma_{\text {in }}$ is here the steady-state reflection coefficient as seen from the antenna feed-line, and it is thus affected by the reflections over the dielectric interface. Considering the free-space reflection coefficient $\Gamma_{a}$ at the antenna input port, $\Gamma_{\text {in }}$ can be written as

$$
\Gamma_{\text {in }}=\Gamma_{a}+\alpha \Gamma_{d}
$$


where $\alpha$ is related to the transmitting properties of the antenna [14]; the finite directivity of the antenna implies that $|\alpha|<1$. These considerations lead to the following result:

$$
\frac{1}{P_{R}}\left|\frac{\partial P_{R}}{\partial \tilde{\epsilon}}\right|=\frac{2\left|\alpha \Gamma_{\text {in }}\right|}{1-\left|\Gamma_{\text {in }}\right|^{2}}\left|\frac{\partial \Gamma_{d}}{\partial \tilde{\epsilon}}\right|
$$

and finally to

$$
\frac{1}{P_{R}}\left|\frac{\partial P_{R}}{\partial \tilde{\epsilon}}\right|<\frac{\left|\Gamma_{\text {in }}\right|}{1-\left|\Gamma_{\text {in }}\right|^{2}} \frac{1}{\left|\chi(1+\chi)^{2}\right|} .
$$

Therefore, the relative sensitivity of the power radiated by the antenna is approximately bounded by $\left|\Gamma_{\text {in }}\right| /|\chi|^{3}$, which is at least one order of magnitude smaller than the signalenergy sensitivity. This result proves that, unless in a very near-field configuration, the power radiated by the antenna is less strongly affected by changes in the lossy half-space characteristics than the TDP, as reported in [7].

\section{CONCLUSIONS}

We have introduced a spectral approach for the analysis of the sensitivity of the TDP to drifts of the electrical properties of a lossy half-space. The definition of a sensitivity operator and of the derivative matrices has shown that their eigenvalues distribution leads to a clear understanding of complex phenomena, such as the dispersiveness of the TDP sensitivity and in particular the fact that the sensitivity to the conductivity is more critical. The same approach was extended to the case of a stratified structure, in order to investigate how a lossless shell modifies the TDP and its sensitivity.

In all these scenarios, the fundamental role played by the reactive content of the source PWS was highlighted, pointing out how it gives rise to a statistically dispersive behaviour of the TDP and its sensitivity to drifts in the electrical parameters of the lossy medium. Hence, the very idea of characterizing the sensitivity in a deterministic way, independently from the source, is not physically sound, especially for near-field sources. These results should thus lead to a better understanding of the phenomena involved in near-field configurations, such as in SAR applications.

\section{APPENDIX I}

\section{DEFINITION OF THE SPECTRAL TRANSMISSION OPERATOR AND RELATED DERIVATIVES}

The transmission and the reflection operators, respectively $\Pi$ and $\boldsymbol{\Gamma}$, for a dielectric interface between two media are defined as [5]:

$$
\begin{aligned}
\boldsymbol{\Pi} & =2 \mathbf{X}^{-1}\left(\mathbf{Y}_{1}+\mathbf{Y}_{2}\right)^{-1} \mathbf{Y}_{1} \mathbf{X} \\
\boldsymbol{\Gamma} & =\mathbf{X}^{-1}\left(\mathbf{Y}_{1}+\mathbf{Y}_{2}\right)^{-1}\left(\mathbf{Y}_{1}-\mathbf{Y}_{2}\right) \mathbf{X} \\
\mathbf{Y}_{i} & =-\frac{1}{\omega \mu_{0} k_{z, i}}\left[\begin{array}{cc}
k_{i}^{2}-k_{x}^{2} & -k_{x} k_{y} \\
-k_{x} k_{y} & k_{i}^{2}-k_{y}^{2}
\end{array}\right] \\
\mathbf{X} & =\left[\begin{array}{cc}
0 & 1 \\
-1 & 0
\end{array}\right],
\end{aligned}
$$

being $k_{z, i}=\sqrt{k_{i}^{2}-\|\mathbf{K}\|^{2}}, i \in[1,2]$.
By applying the derivative chain-rule, the first derivative $\partial \Pi / \partial \tilde{\epsilon}$ is given by:

$$
\frac{\partial \boldsymbol{\Pi}}{\partial \tilde{\epsilon}}=\frac{\partial \boldsymbol{\Pi}}{\partial k_{2}} \frac{\partial k_{2}}{\partial \tilde{\epsilon}}
$$

where

$$
\begin{aligned}
\frac{\partial \mathbf{\Pi}}{\partial k_{2}} & =-2 \mathbf{X}^{-1} \mathbf{B} \frac{\partial \mathbf{Y}_{2}}{\partial k_{2}} \mathbf{B} \mathbf{Y}_{1} \mathbf{X} \\
\mathbf{B} & =\left(\mathbf{Y}_{1}+\mathbf{Y}_{2}\right)^{-1} \\
\frac{\partial \mathbf{Y}_{2}}{\partial k_{2}} & =-\frac{k_{2}}{k_{z, 2}}\left[\frac{\mathbf{Y}_{2}}{k_{z, 2}}+\frac{2}{\omega \mu_{0}} \mathbf{1}\right] \\
\frac{\partial k_{2}}{\partial \tilde{\epsilon}} & =\frac{k_{1}}{2 \sqrt{\tilde{\epsilon}}} .
\end{aligned}
$$

In the same way, the second derivative is given by:

$$
\frac{\partial^{2} \boldsymbol{\Pi}}{\partial \tilde{\epsilon}^{2}}=\frac{\partial^{2} \boldsymbol{\Pi}}{\partial k_{2}^{2}}\left(\frac{\partial k_{2}}{\partial \tilde{\epsilon}}\right)^{2}+\frac{\partial \boldsymbol{\Pi}}{\partial k_{2}} \frac{\partial^{2} k_{2}}{\partial \tilde{\epsilon}^{2}},
$$

where

$$
\begin{aligned}
\frac{\partial^{2} \mathbf{\Pi}}{\partial k_{2}{ }^{2}} & =2 \mathbf{X}^{-1} \mathbf{B}\left(2 \frac{\partial \mathbf{Y}_{2}}{\partial k_{2}} \mathbf{B} \frac{\partial \mathbf{Y}_{2}}{\partial k_{2}}+\right. \\
& \left.-\frac{\partial^{2} \mathbf{Y}_{2}}{\partial k_{2}{ }^{2}}\right) \mathbf{B} \mathbf{Y}_{1} \mathbf{X} \\
\frac{\partial^{2} \mathbf{Y}_{2}}{\partial k_{2}{ }^{2}} & =\mathbf{Y}_{2} \frac{\|\mathbf{K}\|^{2}}{k_{z, 2}^{4}}+\mathbf{1} \frac{2}{\omega \mu_{0} k_{z, 2}}\left(2 \frac{\|\mathbf{K}\|^{2}}{k_{z, 2}^{2}}+1\right) \\
\frac{\partial^{2} k_{2}}{\partial \tilde{\epsilon}^{2}} & =-\frac{k_{1}}{4 \tilde{\epsilon}^{3 / 2}} .
\end{aligned}
$$

Concerning the derivatives of the $\mathbf{T}$ operator, we get:

$$
\frac{\partial \mathbf{T}}{\partial \tilde{\epsilon}}=\left[\begin{array}{cc}
0 & 0 \\
0 & 0 \\
k_{x} & k_{y}
\end{array}\right] \frac{1}{2} \frac{k_{1}^{2}}{k_{z, 2}^{3}}
$$

and

$$
\frac{\partial^{2} \mathbf{T}}{\partial \tilde{\epsilon}^{2}}=-\left[\begin{array}{cc}
0 & 0 \\
0 & 0 \\
k_{x} & k_{y}
\end{array}\right] \frac{3}{4} \frac{k_{1}^{4}}{k_{z, 2}^{5}}
$$

\section{APPENDIX II}

PROOF OF (25)

The sensitivity operator defined in (18) operates over a matrix $\mathbf{A} \in \mathbb{C}^{2 \times 2}$. We consider at first the fact that any matrix can be decomposed into the sum of an Hermitian part $\mathbf{A}_{H}$ and a skew-Hermitian one $\mathbf{A}_{S}$. Hence, the matrix $\mathbf{A}_{H}$ is orthonormal, i.e., with eigenvalues $\lambda_{H i} \in \mathbb{R}$, as well as $\mathrm{j} \mathbf{A}_{S}$; this last claim implies that the eigenvalues $\lambda_{S i}$ of $\mathbf{A}_{S}$ are purely imaginary. Furthermore, thanks to their symmetry properties the diagonalization matrices are orthonormal too, so that one can write:

$$
\begin{aligned}
\mathbf{A}_{H} & =\mathbf{X}_{H} \boldsymbol{\Lambda}_{H} \mathbf{X}_{H}^{\mathrm{H}} \\
\mathbf{A}_{S} & =\mathbf{X}_{S} \boldsymbol{\Lambda}_{S} \mathbf{X}_{S}^{\mathrm{H}},
\end{aligned}
$$

where $\boldsymbol{\Lambda}_{H, S}$ are diagonal matrices containing the eigenvalues of, respectively, the Hermitian and the skew-Hermitian component of $\mathbf{A}$. Imposing $\mathbf{v}=\hat{\mathbf{E}}_{\mathrm{tx}}^{\|}$, we are able to write the 
integrand of the numerator of (18) as:

$$
\begin{aligned}
\mathbf{v}^{\mathrm{H}} \mathbf{A} \mathbf{v} & =\mathbf{e}_{H}^{\mathrm{H}} \boldsymbol{\Lambda}_{H} \mathbf{e}_{H}+\mathbf{e}_{S}^{\mathrm{H}} \boldsymbol{\Lambda}_{S} \mathbf{e}_{S} \\
& =\sum_{i} \lambda_{H i}\left|\mathbf{e}_{H i}\right|^{2}+\sum_{i} \lambda_{S i}\left|\mathbf{e}_{S i}\right|^{2},
\end{aligned}
$$

having introduced the representation of the vector $\mathbf{v}$ into the new basis given by the diagonalization matrices $\mathbf{A}_{H}$ and $\mathbf{A}_{S}$ as:

$$
\begin{aligned}
\mathbf{e}_{H} & =\mathbf{X}_{H}^{\mathrm{H}} \mathbf{v} \\
\mathbf{e}_{S} & =\mathbf{X}_{S}^{\mathrm{H}} \mathbf{v},
\end{aligned}
$$

where the terms $\lambda_{H i}$ and $\lambda_{S i}$ are the eigenvalues of, respectively, matrices $\mathbf{A}_{H}$ and $\mathbf{A}_{S}$.

As shown in Section $\mathrm{V}$, matrix $\mathbf{T}^{\mathrm{H}} \mathbf{T} \simeq \mathbf{1}$ for any source with $k_{\mathrm{BW}} \lesssim k_{0}|\chi|$. Recalling that $\mathbf{X}_{H} \mathbf{X}_{H}^{\mathrm{H}}=\mathbf{X}_{S} \mathbf{X}_{S}^{\mathrm{H}}=\mathbf{1}$, we can state that

$$
\mathbf{v}^{\mathrm{H}} \mathbf{T}^{\mathrm{H}} \mathbf{T} \mathbf{v} \simeq \sum_{i}\left|\mathbf{e}_{H i}\right|^{2}=\sum_{i}\left|\mathbf{e}_{S i}\right|^{2}
$$

leading to

$$
\begin{aligned}
S(\mathbf{A}) & \simeq \sum_{i} \int \lambda_{H i} \frac{\left|\mathbf{e}_{H i}\right|^{2}}{\sum_{m} \int\left|\mathbf{e}_{H m}\right|^{2} \mathrm{~d} \mathbf{K}} \mathrm{d} \mathbf{K}+ \\
& +\sum_{i} \int \lambda_{S i} \frac{\left|\mathbf{e}_{S i}\right|^{2}}{\sum_{m} \int\left|\mathbf{e}_{S m}\right|^{2} \mathrm{~d} \mathbf{K}} \mathrm{d} \mathbf{K}
\end{aligned}
$$

In order to compute the statistical average of $S(\mathbf{A})$, the following functions need to be studied

$$
\frac{w_{i}}{2}=\frac{\left|\mathbf{e}_{i}\right|^{2}}{\sum_{m} \int\left|\mathbf{e}_{m}\right|^{2} \mathrm{~d} \mathbf{K}},
$$

where e stands for $\mathbf{e}_{H}$ or $\mathbf{e}_{S}$. This function represents the energy distribution of the PWS normalized to its total energy; it can thus be regarded as a function describing an envelope. Only one component of the PWS, as represented over the diagonalized basis, is considered, according to the value of the index $i$. It is reasonable to assume that the two components of this function are identically distributed; therefore, their averages are identical too. Hence, the energy-density spectrum $\langle w\rangle=\left\langle w_{i}\right\rangle, \forall i$ can be defined, yielding

$$
\langle S(\mathbf{A})\rangle \simeq \int\left[\bar{\lambda}_{H}(\mathbf{K})+\bar{\lambda}_{S}(\mathbf{K})\right]\langle w(\mathbf{K})\rangle \mathrm{d} \mathbf{K} \quad,
$$

where $\bar{\lambda}_{H}$ and $\bar{\lambda}_{S}$ are the arithmetic means of, respectively, the eigenvalues of matrices $\mathbf{A}_{H}$ and $\mathbf{A}_{S}$. The fact that the same envelope has been used for the Hermitian and the skew-Hermitian parts is due to the unitary property of the diagonalization matrices. The energy content of the PWS is therefore not modified passing from one basis to the other. No assumption has been made in order to come to this result; it is therefore independent of the probability distribution of the energy-density spectrum.

\section{REFERENCES}

[1] A. Baños, Dipole Radiation in the Presence of a Conducting Halfspace, Pergamon, 1966.

[2] K.L. Carr, "Antenna: the critical element in successful medical technology," Microwave Symposium Digest, 1990., IEEE MTT-S International, 8-10 May 1990.

[3] N. Kuster, Q. Balzano, "Energy absorption mechanism by biological bodies in the near field of dipole antennas above $300 \mathrm{MHz}$," IEEE Transactions on Vehicular Technology, Vol. 41, Issue 1, Febraury 1992.

[4] M.G. Douglas, C.-K. Chou, "Enabling the use of broadband tissue equivalent liquids for specific absorption rate measurements," IEEE International Symposium on Electromagnetic Compatibility, EMC 2007, 9-13 July 2007.

[5] C. Scott, The Spectral Domain Method in Electromagnetics, Artech House, 1989.

[6] J.E. Hansen, Spherical Near-Field Antenna Measurements, IEE Electromagnetic Waves Series 26, 1988.

[7] L.M. Correia, "Mobile Broadband Multimedia Networks: Techniques, Models and Tools for 4G," Academic Press, June 2006.

[8] D.A. Harville, Matrix algebra from a statistician's perspective, Springer, 2000.

[9] International Electrotechnical Commission, "Human Exposure to Radio Frequency Fields From Handheld and Body-Mounted Wireless Communication Devices - Human Models, Instrumentation and Procedures, Part 2: Procedure to Determine the Specific Absorption Rate (SAR) in the Head and Body for $30 \mathrm{MHz}$ to $6 \mathrm{GHz}$ Handheld and Body-Mounted Devices Used in Close Proximity to the Body," IEC 62209, pt. 2, June 2008 (committee draft).

[10] D.R. Rhodes, "On a fundamental principle in the theory of planar antennas," Proceedings of the IEEE, Vol. 52, Issue 9, September 1964.

[11] G.H. Golub, C.F. Van Loan, Matrix Computations, The John Hopkins University Press, Third Edition, 1996.

[12] T. Onishi, S. Uebayshi, "Influence of phantom shell on SAR measurement in 3-6 GHz frequency range," IEICE Trans. Commun., Vol. E88-B, No.8, August 2005.

[13] A. Christ, A. Klingenböck, T. Samaras, C. Goiceanu, N. Kuster, "The dependence of electromagnetic far-field absorption on body tissue composition in the frequency range from $300 \mathrm{MHz}$ to $6 \mathrm{GHz}$," IEEE Transactions on Microwave Theory and Techniques, vol. 54, No. 5, May 2006.

[14] D.M. Kerns, Plane-Wave Scattering-Matrix Theory of Antennas and Antenna-Antenna Interactions, National Bureau of Standards, 1981. 